PROCEEDINGS OF THE

AMERICAN MATHEMATICAL SOCIETY

Volume 129, Number 12, Pages 3741-3747

S 0002-9939(01)06002-6

Article electronically published on May 21, 2001

\title{
A WEAK ASPLUND SPACE WHOSE DUAL IS NOT WEAK* FRAGMENTABLE
}

\author{
PETAR S. KENDEROV, WARREN B. MOORS, AND SCOTT SCIFFER
}

(Communicated by Jonathan M. Borwein)

\begin{abstract}
Under the assumption that there exists in the unit interval $[0,1]$ an uncountable set $A$ with the property that every continuous mapping from a Baire metric space $B$ into $A$ is constant on some non-empty open subset of $B$, we construct a Banach space $X$ such that $\left(X^{*}\right.$, weak*) belongs to Stegall's class but $\left(X^{*}\right.$, weak $\left.^{*}\right)$ is not fragmentable.
\end{abstract}

\section{INTRODUCTION}

We say that a Banach space $X$ is weak Asplund if every continuous convex function defined on a non-empty open convex subset $A$ of $X$ is Gâteaux differentiable at the points of a residual subset of $A$. In the study of weak Asplund spaces Stegall 8 introduced the following class of topological spaces, which are defined in terms of minimal uscos. Recall that a set-valued mapping $\varphi: X \rightarrow 2^{Y}$ acting between topological spaces $X$ and $Y$ is called an usco mapping if for each $x \in X, \varphi(x)$ is a non-empty compact subset of $Y$ and for each open set $W$ in $Y,\{x \in X: \varphi(x) \subseteq W\}$ is open in $X$. An usco mapping $\varphi: X \rightarrow 2^{Y}$ is called minimal if its graph does not properly contain the graph of any other usco defined on $X$. We say that a topological space $Y$ belongs to Stegall's $\operatorname{class}(\mathcal{S})$ if for every Baire space $B$ and minimal usco $\varphi: B \rightarrow 2^{Y}, \varphi$ is single-valued at the points of a residual subset of $B$. In [8] Stegall showed that a Banach space $X$ is weak Asplund if $\left(X^{*}\right.$, weak $\left.{ }^{*}\right)$ lies in class $(\mathcal{S})$. In fact, Stegall proved that if the dual unit ball $B_{X^{*}}$ of $X$ equipped with the weak* topology belongs to $\operatorname{class}(\mathcal{S})$, then $X$ is weak Asplund. Another class of topological spaces that have played a significant role in the study of weak Asplund spaces is the class of fragmentable spaces. We say that a topological space $Y$ is fragmented by a pseudo metric $\rho$ if every non-empty subset of $Y$ contains a non-empty relatively open set of arbitrarily small $\rho$-diameter. A space that is fragmented by some metric is called fragmentable. An easy argument shows that fragmentable spaces belong to $\operatorname{Stegall's~} \operatorname{class}(\mathcal{S})$ (see Theorem 5.1.11 in $[2$ ). The converse question was considered in [4]. Indeed, in that paper the author shows

Received by the editors February 17, 2000 and, in revised form, April 22, 2000.

2000 Mathematics Subject Classification. Primary 54C60, 46B20, 54C10.

Key words and phrases. Stegall's class, fragmentability, weak Asplund space, double arrow space, Baire space, minimal usco.

The first author was partially supported by Grant MM-701/97 of the National Fund for Scientific Research of the Bulgarian Ministry of Education, Science and Technology.

The second author was supported by a Marsden fund grant, VUW 703, administered by the Royal Society of New Zealand. 
that under some additional set-theoretic assumptions there are compact spaces in Stegall's class $(\mathcal{S})$ that are not fragmentable. We show in this paper that under similar set-theoretic assumptions there are Banach spaces $X$ such that $\left(X^{*}\right.$, weak $\left.{ }^{*}\right)$ lies in $\operatorname{Stegall's~class~}(\mathcal{S})$ but $\left(X^{*}\right.$, weak $\left.^{*}\right)$ is not fragmentable.

\section{Construction of a Banach space}

Given a subset $A$ of $(0,1)$ we shall consider the Banach space $D_{A}$ of all realvalued functions on $(0,1]$ that have finite right-hand limits at the points of $[0,1)$, are left-continuous at the points of $(0,1]$ and are continuous at the points of $(0,1] \backslash A$, endowed with sup-norm. Then we shall characterise the duals of these spaces in terms of functions of bounded variation. Given bounded functions $f$ and $\alpha$ defined on $(0,1]$ and $[0,1]$ respectively and a partition $P:=\left\{t_{k}: 0 \leq k \leq n\right\}$ of $[0,1]$ where

$$
0=t_{0}<t_{1}<t_{2}<\cdots<t_{n}=1
$$

the Riemann-Stieltjes sum of $f$ with respect to $\alpha$, determined by $P$, is the real number

$$
S(P, f, \alpha):=\sum_{k=1}^{n} f\left(t_{k}\right) \cdot\left[\alpha\left(t_{k}\right)-\alpha\left(t_{k-1}\right)\right]
$$

We say that $f$ is Riemann-Stieltjes integrable with respect to $\alpha$ if there exists a real number $I$ such that for every $\varepsilon>0$ there exists a partition $P_{\varepsilon}$ of $[0,1]$ such that $|S(P, f, \alpha)-I|<\varepsilon$ for all partitions $P$ that refine $P_{\varepsilon}$. In this case $I$ is denoted by $I:=\int_{[0,1]} f(t) \mathrm{d} \alpha(t)$ and is called the Riemann-Stieltjes integral of $f$ with respect to $\alpha$.

For any subset $A$ of $(0,1)$ we shall denote by $B V_{A}[0,1]$ the space of all realvalued functions of bounded variation on $[0,1]$ that are right-continuous at the points of $(0,1) \backslash A$ and map 0 to 0 . We will consider this space endowed with the total variation norm, i.e. for each $\alpha \in B V_{A}[0,1]$

$$
\|\alpha\|:=\operatorname{Var}(\alpha)=\sup \left\{\sum_{k=1}^{n}\left|\alpha\left(t_{k}\right)-\alpha\left(t_{k-1}\right)\right|:\left\{t_{k}: 0 \leq k \leq n\right\} \text { is a partition of }[0,1]\right\}
$$

The proof of the following lemma is straightforward.

Lemma 1 (Uniform approximation lemma). Let $A$ be any dense subset of $(0,1)$, $f \in D_{A}$ and $\varepsilon>0$. Then there exists a partition $P_{\varepsilon}:=\left\{t_{k}: 0 \leq k \leq n\right\}$ of $[0,1]$ with $t_{k} \in A$ for all $1 \leq k<n$ such that $\left\|f-f_{P_{\varepsilon}}\right\|_{\infty}<\varepsilon$, where $f_{P_{\varepsilon}}:(0,1] \rightarrow \mathbb{R}$ is defined by $f_{P_{\varepsilon}}(t):=\sum_{k=1}^{n} f\left(t_{k}\right) \cdot \chi_{\left(t_{k-1}, t_{k}\right]}(t)$.

One can now use the previous lemma to prove the following theorem.

Theorem 1. Suppose that $\alpha:[0,1] \rightarrow \mathbb{R}$ has bounded variation and $f \in D_{(0,1)}$. Then $f$ is Riemann-Stieltjes integrable with respect to $\alpha$.

Proof. First note that to show $f$ is Riemann-Stieltjes integrable with respect to $\alpha$ we need only show that for every $\varepsilon>0$ there exists a partition $P_{\varepsilon}$ of $[0,1]$ such that $\left|S\left(P_{\varepsilon}, f, \alpha\right)-S\left(P^{\prime}, f, \alpha\right)\right|<\varepsilon$ for all partitions $P^{\prime}$ that refine $P_{\varepsilon}$. Further, an elementary calculation shows that for any $g, g^{\prime} \in D_{(0,1)}$ and partition $P$ we have 
that $\left|S(P, g, \alpha)-S\left(P, g^{\prime}, \alpha\right)\right| \leq\left\|g-g^{\prime}\right\| \cdot \operatorname{Var}(\alpha)$. Therefore, if we fix $\varepsilon>0$ and choose a partition $P$ of $[0,1]$ such that $\left\|f-f_{P}\right\|<\varepsilon /(\operatorname{Var}(\alpha)+1)$, then

$$
\begin{aligned}
\left|S(P, f, \alpha)-S\left(P^{\prime}, f, \alpha\right)\right| & \leq\left|S(P, f, \alpha)-S\left(P, f_{P}, \alpha\right)\right| \\
& +\left|S\left(P, f_{P}, \alpha\right)-S\left(P^{\prime}, f_{P}, \alpha\right)\right| \\
& +\left|S\left(P^{\prime}, f_{P}, \alpha\right)-S\left(P^{\prime}, f, \alpha\right)\right| \\
& <0+0+\varepsilon=\varepsilon
\end{aligned}
$$

for all partitions $P^{\prime}$ that refine $P$.

By a slight adaption of the proof of Riesz's representation theorem for the dual of $\left(C[0,1],\|\cdot\|_{\infty}\right)$ we can obtain the following representation theorem. Note: it is easiest to make the adaption to the proof of Riesz's representation theorem that relies upon the Hahn-Banach extension theorem. In fact the standard proof only uses extensions to the space $D_{(0,1)}$ and not to all of $B[0,1]$ - the space of bounded functions on $[0,1]$; see [1]. Further details may also be found in the paper [6].

Theorem 2. Let $A$ be any subset of $(0,1)$. Then the dual of $D_{A}$ is isometrically isomorphic to $B V_{A}[0,1]$. In particular the mapping $T: B V_{A}[0,1] \rightarrow D_{A}^{*}$ defined by $T(\alpha)(x):=\int_{[0,1]} x(t) \mathrm{d} \alpha(t)$ for each $x \in D_{A}$ is an isometry from $B V_{A}[0,1]$ onto $D_{A}^{*}$.

For a non-empty subset $A$ of $[0,1]$ we shall denote by $\tau_{A}$ the topology (on $\left.B V_{A}[0,1]\right)$ of pointwise convergence on $A \cup\{1\}$. If $A$ is dense in $[0,1]$, then $\tau_{A}$ is a Hausdorff topology. Moreover, the closed unit ball in $B V_{A}[0,1]$ (with respect to the total variation norm) is $\tau_{A}$-compact.

Corollary 1. For a non-empty subset $A$ of $(0,1),\left(B V_{A}[0,1], \tau_{A}\right)$ is homeomorphic to $D_{A}^{*}$ endowed with the weak topology generated by the functions $\chi_{(0, a]}$ with $a \in$ $A \cup\{1\}$. If $A$ is dense in $(0,1]$, then $\tau_{A}$ is Hausdorff and the closed unit ball $B_{B V_{A}[0,1]}$ in $B V_{A}[0,1]$ with the $\tau_{A}$-topology is homeomorphic to $\left(B_{D_{A}^{*}}\right.$, weak $\left.k^{*}\right)$. In fact the mapping $T$ defined in the previous theorem, restricted to the ball $B_{B V_{A}[0,1]}$, realizes such a homeomorphism.

Proof. The proof of the first assertion is based upon the simple fact that for each $\alpha \in B V_{A}[0,1]$ and $t \in A \cup\{1\}, T(\alpha)\left(\chi_{(0, t]}\right)=\alpha(t)$. The fact that $T$ restricted to $B_{B V_{A}[0,1]}$ realizes a homeomorphism onto $\left(B_{D_{A}^{*}}\right.$, weak $\left.{ }^{*}\right)$ follows from the fact that on $B_{D_{A}^{*}}$ the relative weak* topology and the relative topology generated by the functions $\chi_{(0, t]}, t \in A \cup\{1\}$ coincide (see Lemma 1 ).

\section{3. $\left(B V_{A}[0,1], \tau_{A}\right)$ BELONGS TO $\operatorname{CLASS}(\mathcal{S})$}

We begin this section with the following preliminary theorem.

Theorem 3. Let $Y$ be a compact topological space and $\rho$ a metric on it. Then $Y$ belongs to class $(\mathcal{S})$ if (and only if) for $\varepsilon>0$, each Baire metric space $B$ and each minimal usco $\varphi: B \rightarrow 2^{Y}$ there exists a point $x \in B$ such that $\rho$-diam $\varphi(x) \leq \varepsilon$.

Proof. By the "factorization theorem" in 5] we need only show that for every complete metric space $M$ and minimal usco $\varphi: M \rightarrow 2^{Y}$ there exists a residual set $R$ of $M$ such that $\varphi$ is single-valued at the points of $R$. If we now apply the proof of Theorem 3.2.6 in 2 to our current situation we obtain the desired result. 
Lemma 2. Let $\varphi: X \rightarrow 2^{Y}$ be a minimal usco acting between topological spaces $X$ and $Y$ and let $f: Y \rightarrow \mathbb{R}$ be a continuous function. Then there is a residual set $R$ in $X$ such that the composition mapping $f \circ \varphi: X \rightarrow 2^{\mathbb{R}}$ defined by $(f \circ \varphi)(x):=$ $\{f(y): y \in \varphi(x)\}$ is single-valued at the points of $R$.

Proof. By Lemma 3.1.2(iv) in [2], $f \circ \varphi$ is a minimal usco on $X$ and so the result follows from Theorem 5.1.11 in [2].

In the remainder of this section $A$ will always denote a dense subset of $(0,1)$ that satisfies the property: $(*)$ Every continuous function from a Baire metric space $B$ into $A$ is constant on some non-empty open subset of $B$.

Of course every countable dense subset of $(0,1)$ has this property; however we shall be particularly interested in the case when $A$ is uncountable, if indeed such a set exists.

Theorem 4. Let $A$ be a dense subset of $(0,1)$ that satisfies property $(*)$. Then $\left(B V_{A}[0,1], \tau_{A}\right)$ belongs to class $(\mathcal{S})$.

Proof. First, let us note that by Theorem 3.1.5, part(iv) in [2], we need only show that the closed unit ball $B_{B V_{A}[0,1]}$ of $B V_{A}[0,1]$ belongs to $\operatorname{class}(\mathcal{S})$. In fact, we need only show that the $\left(\tau_{A}\right.$-compact) set $M_{A}[0,1]$ of all non-decreasing functions in $B_{B V_{A}[0,1]}$, endowed with the $\tau_{A}$-topology lies in Stegall's class $(\mathcal{S})$. Since if $M_{A}[0,1] \in \operatorname{class}(\mathcal{S})$, then by Theorem 3.1.5, $\operatorname{part}\left(\right.$ iii) in [2], $M_{A}[0,1] \times$ $M_{A}[0,1] \in \operatorname{class}(\mathcal{S})$. However, by the Jordan decomposition theorem $B_{B V_{A}[0,1]} \subseteq$ $\Delta\left(M_{A}[0,1] \times M_{A}[0,1]\right)$, where $\Delta: M_{A}[0,1] \times M_{A}[0,1] \rightarrow B V_{A}[0,1]$ is defined by $\Delta(f, g):=f-g$. Hence the result follows from Theorem 3.1.5, part(i) in [2], since $\Delta$ is a perfect mapping. For any $\alpha, \beta$ in $M_{A}[0,1]$ we define

$$
\begin{aligned}
\rho_{1}(\alpha, \beta) & :=|(\alpha-\beta)(1)|, \quad \rho_{I}(\alpha, \beta):=\int_{0}^{1}|(\alpha-\beta)(t)| \mathrm{d} t, \\
\rho_{J}(\alpha, \beta) & :=\sum_{t \in A}\left|(\alpha-\beta)\left(t^{+}\right)-(\alpha-\beta)(t)\right| .
\end{aligned}
$$

Note: $\left\{t \in A:\left|(\alpha-\beta)\left(t^{+}\right)-(\alpha-\beta)(t)\right|>0\right\}$ is at most countable. Then we define $\rho(\alpha, \beta):=\rho_{1}(\alpha, \beta)+\rho_{I}(\alpha, \beta)+\rho_{J}(\alpha, \beta)$. With a little thought it should be clear that $\rho$ defines a metric on the set $M_{A}[0,1]$. We now proceed via Theorem 3 . To this end, let $\varepsilon>0, B$ be a Baire metric space and $\varphi: B \rightarrow 2^{M_{A}[0,1]}$ be a minimal usco.

Step 1. It is not too difficult to check that $\rho_{I}$ is a continuous pseudo-metric on $M_{A}[0,1]$, i.e. for each $\alpha \in M_{A}[0,1]$ and $r>0$ the set $\left\{\beta \in M_{A}[0,1]: \rho_{I}(\alpha, \beta)<r\right\}$ is $\tau_{A}$-open in $M_{A}[0,1]$. Hence it follows that $\rho_{I}$ "fragments" $M_{A}[0,1]$. It is also very easy to see that $\rho_{1}$ is a continuous pseudo-metric on $M_{A}[0,1]$ and so $\rho_{1}$ also "fragments" $M_{A}[0,1]$. In particular this means that there is a residual set $R \subseteq B$ such that both $\rho_{1}$-diam $\varphi(x)=0$ and $\rho_{I}$-diam $\varphi(x)=0$ at each point $x \in R$ (see the proof of Theorem 5.1.11 in [2]). Therefore by restricting $\varphi$ to $R$ and re-labeling we may assume, without loss of generality, that both $\rho_{1}$-diam $\varphi(x)=0$ and $\rho_{I^{-}}$diam $\varphi(x)=0$ for all $x \in B$. One immediate consequence of this is that for each $x \in B$ we may unambiguously refer to the left-hand and right-hand limits of $\varphi(x)$, since if $\alpha, \beta \in \varphi(x)$, then both the left-hand and right-hand limits of $\alpha$ and $\beta$ coincide on $[0,1]$. 
Step 2. In this step we decompose the space $M_{A}[0,1]$ into countably many parts, $\left\{M_{m, n,(F, f)}:(m, n,(F, f)) \in \mathbb{N}^{2} \times \mathcal{F}\right\}$, but first we introduce some notation. For each $\alpha \in M_{A}[0,1]$ and $m \in \mathbb{N}$,

$$
\begin{aligned}
S(\alpha, m) & :=\left\{t \in A: \alpha\left(t^{+}\right)-\alpha\left(t^{-}\right)>1 / m\right\} \quad \text { and } \\
L^{1}(\alpha, m) & :=\sum_{t \in S(\alpha, m)}\left[\alpha\left(t^{+}\right)-\alpha\left(t^{-}\right)\right] .
\end{aligned}
$$

The notation $S(\alpha, \infty)$ and $L^{1}(\alpha, \infty)$ will have the expected meaning. For each $m \in \mathbb{N}$ we define, $M_{m}:=\left\{\alpha \in M_{A}[0,1]: L^{1}(\alpha, m)>L^{1}(\alpha, \infty)-\varepsilon / 2\right\}$ and for each partition $P:=\left\{t_{k}: 0 \leq k \leq n\right\}$ of $[0,1]$ we let $I_{k}(P):=\left[t_{k-1}, t_{k}\right], 1 \leq k \leq n$. Then for each $n \in \mathbb{N}$ we let $P_{n}$ denote the uniform $1 / n$-partition of $[0,1]$ and we define

$$
\begin{aligned}
M_{m, n}:=\left\{\alpha \in M_{m}: P_{n} \cap\right. & S(\alpha, m)=\emptyset \text { and } \\
& \left.\quad \operatorname{card}\left[S(\alpha, m) \cap I_{k}\left(P_{n}\right)\right] \leq 1 \text { for } k \in\{1,2, \cdots, n\}\right\} .
\end{aligned}
$$

One can check that $\bigcup\left\{M_{m, n}:(m, n) \in \mathbb{N}^{2}\right\}=M_{A}[0,1]$. Now, with $m$ and $n$ fixed we further decompose $M_{A}[0,1]$ as follows: For each fixed non-empty subset $F \subseteq\{1,2, \cdots, n\}$ and function $f: F \rightarrow \mathbb{Q}^{2}$, i.e. $f(k):=\left(f_{1}(k), f_{2}(k)\right) \in \mathbb{Q}^{2}$, we consider the set

$$
\begin{aligned}
M_{m, n,(F, f)}:=\left\{\alpha \in M_{m, n}\right. & : \operatorname{card}\left[I_{k}\left(P_{n}\right) \cap S(\alpha, m)\right]=1 \text { if, and only if, } k \in F, \\
& \text { and } \max \left\{\left|\alpha\left(t^{-}\right)-f_{1}(k)\right|,\left|\alpha\left(t^{+}\right)-f_{2}(k)\right|\right\}<1 /(4 m) \\
& \text { for each } \left.t \in I_{k}\left(P_{n}\right) \cap S(\alpha, m) \text { and } k \in F\right\} .
\end{aligned}
$$

If we let $\mathcal{F}$ denote the family of all such pairs $(F, f)$, then $\mathcal{F}$ is at most countable. Hence $\left\{M_{m, n,(F, f)}:(m, n,(F, f)) \in \mathbb{N}^{2} \times \mathcal{F}\right\}$ is a countable decomposition of $M_{A}[0,1]$.

Step 3. For any subset $X \subseteq M_{A}[0,1]$ we define $\varphi^{-1}(X):=\{x \in B: \varphi(x) \cap X \neq \emptyset\}$. Now since $M_{A}[0,1]=\bigcup\left\{M_{m, n,(F, f)}:(m, n,(F, f)) \in \mathbb{N}^{2} \times \mathcal{F}\right\}$, it follows that $\bigcup\left\{\varphi^{-1}\left(M_{m, n,(F, f)}\right):(m, n,(F, f)) \in \mathbb{N}^{2} \times \mathcal{F}\right\}=B$. Therefore there must be some $\left(m^{\prime}, n^{\prime},\left(F^{\prime}, f^{\prime}\right)\right) \in \mathbb{N}^{2} \times \mathcal{F}$ such that $\varphi^{-1}\left(M_{m^{\prime}, n^{\prime},\left(F^{\prime}, f^{\prime}\right)}\right)$ is second (Baire) category in $B$. Moreover, since the set $M_{m^{\prime}, n^{\prime},\left(F^{\prime}, f^{\prime}\right)}$ is defined solely in terms of the left-hand and right-hand limits of its members it follows, by the note at the end of Step 1, that

$$
\varphi\left(\varphi^{-1}\left(M_{m^{\prime}, n^{\prime},\left(F^{\prime}, f^{\prime}\right)}\right)\right) \subseteq M_{m^{\prime}, n^{\prime},\left(F^{\prime}, f^{\prime}\right)} .
$$

Further, by Proposition 3.2.5 in [2] there exists a non-empty open set $U$ in $B$ such that $B^{\prime}:=U \cap \varphi^{-1}\left(M_{m^{\prime}, n^{\prime},\left(F^{\prime}, f^{\prime}\right)}\right)$ is dense in $U$ and a Baire space with the relative topology. Now by applying Lemma 2 in [4] twice we see that the restriction of $\varphi$ to $B^{\prime}$ is a minimal usco. In this way, we see that there is no loss of generality in assuming that $\varphi(B) \subseteq M_{m^{\prime}, n^{\prime},\left(F^{\prime}, f^{\prime}\right)}$.

Step 4. For each $k \in F^{\prime} \subseteq\left\{1,2, \cdots, n^{\prime}\right\}$ we define the function $g_{k}: B \rightarrow A$ by $g_{k}(x):=S\left(\varphi(x), m^{\prime}\right) \cap I_{k}\left(P_{n^{\prime}}\right)$. Note: this definition is sensible since for each $x \in B$ and $\alpha, \beta \in \varphi(x), S\left(\alpha, m^{\prime}\right)=S\left(\beta, m^{\prime}\right)$. It now follows from the $\tau_{A}$-upper semi-continuity of $\varphi$ and the definition of $M_{m^{\prime}, n^{\prime},\left(F^{\prime}, f^{\prime}\right)}$ that each $g_{k}$ is continuous on $B$. Hence by property $(*)$ there exists a non-empty open subset $U$ of $B$ such that each $g_{k}, k \in F^{\prime}$, is constant on $U$. 
Step 5. For each $k \in F^{\prime}$ define $t_{k}:=g_{k}(x), x \in U$. Then by Lemma 2 there exists a residual set $R$ in $U$ such that each of the uscos $\hat{t}_{k} \circ \varphi: U \rightarrow 2^{\mathbb{R}}$ defined by $\left(\hat{t}_{k} \circ \varphi\right)(x):=\left\{\alpha\left(t_{k}\right): \alpha \in \varphi(x)\right\}$ are single-valued on $R$. We claim that $\rho$-diam $\varphi(x) \leq \varepsilon$ for each $x \in R$. To see this, first note that it is sufficient to show that $\rho_{J}$-diam $\varphi(x) \leq \varepsilon$ for each $x \in R$. Now fix $x_{0} \in R$ and consider $\alpha, \beta \in \varphi\left(x_{0}\right)$; then

$$
\rho_{J}(\alpha, \beta)=\sum_{t \in A}\left|(\alpha-\beta)\left(t^{+}\right)-(\alpha-\beta)(t)\right|=\sum_{t \in S(\alpha, \infty)}\left|(\alpha-\beta)\left(t^{+}\right)-(\alpha-\beta)(t)\right| .
$$

However, if $t \in S\left(\alpha, m^{\prime}\right)$, then $\left|(\alpha-\beta)\left(t^{+}\right)-(\alpha-\beta)(t)\right|=0$ since (by Step 1) $\alpha\left(t^{+}\right)=\beta\left(t^{+}\right)$and (as just noted) $\alpha(t)=\beta(t)$. On the other hand, if we write $S_{\text {tail }}:=S(\alpha, \infty) \backslash S\left(\alpha, m^{\prime}\right)$, then we have

$$
\begin{aligned}
\sum_{t \in S_{\text {tail }}}\left|(\alpha-\beta)\left(t^{+}\right)-(\alpha-\beta)(t)\right| & \leq \sum_{t \in S_{\text {tail }}} \alpha\left(t^{+}\right)-\alpha(t)+\sum_{t \in S_{\text {tail }}} \beta\left(t^{+}\right)-\beta(t) \\
& \leq \sum_{t \in S_{\text {tail }}} \alpha\left(t^{+}\right)-\alpha\left(t^{-}\right)+\sum_{t \in S_{\text {tail }}} \beta\left(t^{+}\right)-\beta\left(t^{-}\right) \\
& <\varepsilon / 2+\varepsilon / 2=\varepsilon .
\end{aligned}
$$

This shows that $\rho(\alpha, \beta) \leq \varepsilon$ and so $\rho$-diam $\varphi\left(x_{0}\right) \leq \varepsilon$, which completes the proof.

Corollary 2. Let $A$ be any dense subset of $(0,1)$ that satisfies property $(*)$. Then $\left(D_{A}^{*}\right.$, weak $\left.k^{*}\right)$ belongs to $\operatorname{class}(\mathcal{S})$.

We end this section of the paper by returning to the question of the existence of uncountable sets that have property $(*)$. The good news is that there is such a subset $A$ of $(0,1)$ that satisfies property $(*)$ in Gödel's universe $(V=L)$ and hence the set $A^{\prime}:=A \cup[(0,1) \cap \mathbb{Q}]$ will serve our needs; see [7. However, the set $A$ necessarily relies upon additional axioms, as it is known that if we assume the existence of a precipitous ideal over $\omega_{1}$, then for every uncountable separable metric space $A$ there exists a Baire metric space $B$ and a continuous function $f: B \rightarrow A$ such that $\operatorname{int}\left(f^{-1}(a)\right)=\emptyset$ for each $a \in A$ (see [3]).

\section{When is $\left(B V_{A}[0,1], \tau_{A}\right)$ Fragmentable?}

We will show that for every set $A \subseteq(0,1), D_{A}$ is isometrically isomorphic to $C\left(K_{A}\right)$ for some compact Hausdorff space $K_{A}$. Indeed, if $\emptyset \neq A \subseteq(0,1)$, then we may define $K_{A}$ in the following manner: $K_{A}:=[(\{0\} \cup A) \times\{1\}] \cup[(0,1] \times\{0\}]$. We endow $K_{A}$ with the order topology (on $K_{A}$ ) generated by the lexographic (i.e. dictionary) ordering, i.e. $\left(s_{1}, s_{2}\right) \leq\left(t_{1}, t_{2}\right)$ if, and only if, either $s_{1}<t_{1}$ or $s_{1}=t_{1}$ and $s_{2} \leq t_{2}$. It is shown in [4] (see Proposition 2) that $K_{A}$ is always Hausdorff and compact. It is also shown that $K_{A}$ is fragmentable if, and only if, $A$ is countable and this occurs if, and only if, $K_{A}$ is metrizable.

Theorem 5. Let $A$ be a non-empty subset of $(0,1)$. Then $\left(D_{A}^{*}\right.$, weak $\left.k^{*}\right)$ is fragmentable if, and only if, $A$ is countable.

Proof. We define an isometry $T$ from $D_{A}$ onto $C\left(K_{A}\right)$ in the following way: $T(f)((t, 0)):=f(t)$ for all $t \in(0,1]$ and $T(f)((t, 1)):=\lim _{t^{\prime} \rightarrow t^{+}} f\left(t^{\prime}\right)$ for $t \in\{0\} \cup A$. One can check, as in ([2], p. 47), that $T$ is in fact an isometry from $D_{A}$ onto $C\left(K_{A}\right)$. Indeed, it is routine to verify that $T$ is a linear isometry into $C\left(K_{A}\right)$, so it suffices to check that $T$ is surjective. To this end, let $g \in C\left(K_{A}\right)$ and define $f:(0,1] \rightarrow \mathbb{R}$ by $f(t):=g((t, 0))$ for all $t \in(0,1]$. Then $f \in D_{A}$ and $T(f)=g$. 
Corollary 3. If $A$ is an uncountable dense subset of $(0,1)$ that satisfies property $(*)$, then $\left(D_{A}^{*}\right.$, weak $\left.{ }^{*}\right)$ belongs to $\operatorname{class}(\mathcal{S})$ (and so $D_{A}$ is weak Asplund) but $\left(D_{A}^{*}\right.$, weak $\left.^{*}\right)$ is not fragmentable.

\section{REFERENCES}

[1] G. Bachman and L. Narici, Functional Analysis, Academic Press, 1966. MR 36:638

[2] M. Fabian, Gâteaux Differentiability of Convex Functions: Weak Asplund Spaces, John Wiley and Sons, 1997. MR 98h:46009

[3] R. Frankiewicz and K. Kunen, Solution of Kuratowski's problem on functions having the Baire property I, Fund. Math. 128 (1987), 171-180. MR 89a:03090

[4] O. Kalenda, Stegall compact spaces which are not fragmentable, Topology Appl. 96 (1999), 121-132. MR 2000i:54027

[5] P. S. Kenderov and J. Orihuela, A generic factorization theorem, Mathematika 42 (1995), 56-66. MR 96h:54014

[6] W. B. Moors and S. D. Sciffer, Sigma-fragmentable spaces that are not countable unions of fragmentable subspaces, Topology Appl. (to appear.)

[7] I. Namioka and R. Pol, Mappings of Baire spaces into function spaces and Kadec renorming, Israel J. Math. 78 (1992), 1-20. MR 94f:46020

[8] C. Stegall, A class of topological spaces and differentiation of functions on Banach spaces, Vorlesungen aus dem Fachbereich Mathematik der Universität Essen 10 (1983), 63-77. MR 85j:46026

Institute of Mathematics, Bulgarian Academy of Science, Acad. G. Bonchev Street, Block 8, 1113 Sofia, Bulgaria

E-mail address: pkend@bgcict.acad.bg; pkend@math.bas.bg

Department of Mathematics, University of Waikato, Private Bag 3105, Hamilton, New ZeALAND

E-mail address: moors@math.auckland.ac.nz

Department of Mathematics, University of Newcastle, Newcastle NSW-2308, AusTRALIA 